\title{
Two Novel Characterizations of the DE Flip Flop
}

\section{Ali Muhammad Rushdi and Fares Ahmad Ghaleb}

\author{
Department of Electrical and Computer Engineering, King Abdulaziz University, Jeddah 21589, \\ Saudi Arabia \\ arushdi@kau.edu.sa
}

\begin{abstract}
Modern digital circuits, especially those based on large-scale integration devices employ DE flip flops, which are an extension of the D type with the capacity to store an input value only upon request or enabling. The DE flip flop could possibly be described algebraically by its characteristic equation or tabularly by its next-state table (used for analysis purposes) and its excitation table (used for synthesis purposes). This paper explores two novel characterizations of DE flip flops. First, equational and implicational descriptions are presented, and the Modern Syllogistic Method is utilized to produce complete statements of all propositions that are true for a general DE flip flop. Next, methods of Boolean-equation solving are employed to find all possible ways to express the excitations in terms of the present state and next state. The concept of Boolean quotient plays a crucial role in exposing the pertinent concepts and implementing the various desired derivations. This paper is expected to be of an immediate pedagogical benefit, and to facilitate the analysis and synthesis of contemporary sequential digital circuits.

Keywords: Flip flop, Characterization, Modern Syllogistic Method, Boolean-equation solving, Boolean quotient, Excitation.
\end{abstract}

\section{Introduction}

An essential building block of a sequential digital circuit is an elementary memory cell called a flip flop, a one-bit register, a bistable or a bistable multivibrator ${ }^{[1]}$. A flip-flop is a circuit that has two stable states and can be used to store a single bit (binary digit) of data; with one of the two states of the flip flop representing a "one" and the other representing a "zero". A flip flop is usually named in terms of its inputs or excitations. In the following, we use the symbols $y_{i}$ and $Y_{i}$, to denote the present and next states of flip flop number $i$ in a given circuit. We also use upper-case subscripted letters to designate the excitations of a flip flop.
There are many well-known types of flip flops, including those with a single input (such as the $D$ flip flop and the $T$ flip flop), those with two inputs (such as the $S R$ flip flop, the $J K$ flip flop, and the $D E$ flip flop), and those with three or more inputs. Out of these, we single out the $J K$ flip-flop as a very versatile device that was commonly used to construct circuits with discrete components. The $J K$ flip flop is discussed in many publications on digital design (See, e.g., ${ }^{[1-8]}$ ) and its various characterizations are detailed in ${ }^{[1]}$. However, it has been mostly replaced by a variation of the $D$ flip flop, which is conveniently called the $D E$ flip flop (or occasionally, the E-type flip flop $)^{[1,9-12]}$. This variation is simply a $D$ flip flop that is equipped with an enable input $E$. 
This paper is a tutorial exposition about flip flops in general, and about $D E$ flip flops, in particular. After surveying conventional characterizations of $D E$ flip flops (in comparison with those of $J K$ flip flops), we employ novel mathematical methods of logic deduction and Boolean-equation solving for further characterization of the $D E$ flip flop.

The organization of the remainder of this paper is as follows. Section 2 offers an introductory characterization of the $D E$ flip flop in comparison with the $J K$ flip flop. Section 3 uses the Modern Syllogistic Method (MSM) of logic deduction to ferret out all that can be said about the $D E$ flip flop in the most compact form. Section 4 applies methods of 'big' Boolean-equation solving to find all possible solutions of the excitations of the $D E$ flip flop in terms of its present and next states. Section 5 concludes the paper. To make the paper self-contained, it is supplemented with an appendix on the "Boolean Quotient", a crucial concept for some of the current derivations.

\section{The DE Flip Flop Versus the JK Flip Flop}

We recall that the $D E$ flip flop is a variation of the D flip flop, which is simply a D flip flop that is equipped with an enable input $E^{[1,9-12]}$. Despite the contemporary widespread use of the $D E$ flip flop, it is definitely less familiar (to most readers) than the $J K$ flip flop. We devote this section to a brief introduction of the $D E$ flip flop, in comparison to the $J K$ flip flop. Figure 1 presents the characteristic maps of both types of flip flops, in both conventional-map form and variableentered form. Figure 2 displays the transition maps for both types of flip flops. The transition variable $\delta y_{i}$ is a four-valued variable defined to be $\delta y_{i}=0$ when $y_{i}=Y_{i}=$ $0, \delta y_{i}=1$ when $y_{i}=Y_{i}=1, \delta y_{i}=\Delta$ when $y_{i}=0$ and $Y_{i}=1$, and finally $\delta y_{i}=\nabla$ when $y_{i}=1$ and $Y_{i}=0{ }^{[9]}$. Figure 3 presents the excitation maps for both the $J K$ and the $D E$ flip flops. Of course, the excitation map of the $D E$ flip flop (as shown in Fig. 3(b)) is virtually unknown in the literature, but it will be verified and formally derived in the forthcoming sections.

We now digress a little bit to explain why the $J K$ flip flop is superior from the theoretical point of view, and why it was dominantly used in the era of discrete components. A quick glimpse at Fig. 1-3 demonstrates clearly that the $J K$ flip flop has a more balanced behavior and a more versatile capability than the $D E$ flip flop. In fact, the $J K$ flip flop enjoys the following distinctive advantages:

- By contrast to the $S R$ flip-flop, which has an indeterminate outcome when $S_{i}=R_{i}=$ 1 , (or must, otherwise, be operated under the constraint $S_{i} R_{i}=0$ ), the $J K$ flip-flop has the merit of guaranteed determined outcome under unconstrained operation.

- The $J K$ flip-flop is intimately related to other famous flip flops. It is exactly an $S R$ flip flop with an additional external feedback provided via the relations $S_{i}=J_{i} \bar{y}_{i}$ and $R_{i}=K_{i} y_{i}$. These relations ensure the satisfaction of the aforementioned constraint $\left(S_{i} R_{i}=0\right)$ and allow the designer, if he/she wishes, to dispense with external feedback from the outputs of a particular flip flop to its own inputs. The $J K$ flip-flop also combines the delay function of a $D$ flip-flop and the toggle function of a $T$ flip-flop. The $J K$ flip-flop reduces to a $D$ flip-flop by making its excitations complementary $\left(J_{i}=D_{i}, K_{i}=\bar{D}_{i}\right)$, and reduces to a $T$ flip-flop by making its excitations equal $\left(J_{i}=K_{i}=T_{i}\right)$.

- The $J K$ flip-flop possesses the minimum number of excitations that can control its next-state variable to assume any of the four possible values that might be taken by 
a Boolean function of a single variable representing the present-state value, namely: the present state itself, its complement, logic 0 , and logic 1 . In fact the algebraic characteristic equation (next-state equation) of the $J K$ flipflop

$$
Y_{i}=J_{i} \bar{y}_{i} \vee \bar{K}_{i} y_{i},
$$

might be rewritten in the minterm-expansion form

$Y_{i}=\left(y_{i}\right) \bar{J}_{i} \bar{K}_{i} \vee(0) \bar{J}_{i} K_{i} \vee\left(\bar{y}_{i}\right) J_{i} K_{i} \vee(1) J_{i} \bar{K}_{i}$

Equations (1) and (2) can be readily deduced from Fig. 1.

- The excitations of a $J K$ flip-flop can be made independent of its present state by setting them as the Boolean quotients (See Appendix A) ${ }^{[1]}$

$$
\begin{aligned}
J_{i} & =Y_{i} / \bar{y}_{i}, \\
K_{i} & =\bar{Y}_{i} / y_{i} .
\end{aligned}
$$

As stated earlier, a modern digital network, especially one that is based on largescale integration devices (such as a FieldProgrammable Gate Array ( $F P G A)$ ) employs a $D E$ flip flop which is an extension of the $D$ type ${ }^{[1,9-12]}$. This flip flop is similar to a standard $D$ flip flop except that the $D$ input is only enabled when the input $E$ (for "Enable") is equal to logic 1 . When the input $E$ is equal to logic 0 , the flip flop remains in its current state. Hence, the $D E$ flip flop has the capacity to store an input value only upon request or enabling. This behavior differs from that of the $D$ flip flop, which stores a new value (unconditionally) at each active edge of the clock. The characteristic equation for the $D E$ flip flop might be written as

$$
Y_{i}=y_{i} \bar{E}_{i} \vee D_{i} E_{i} \text {. }
$$

Detailed information about the $D E$ flip flop is available in ${ }^{[11]}$, where it is given the designation $E-P E T$ with "PET" standing for
"Positive-Edge-Triggered". Equation (4) might be rewritten as a minterm expansion in the form $Y_{i}=\left(y_{i}\right) \bar{D}_{i} \bar{E}_{i} \vee(0) \bar{D}_{i} E_{i} \vee(1) D_{i} E_{i} \vee\left(y_{i}\right) D_{i} \bar{E}_{i}$

Again, Equations (4) and (5) can be deduced from Fig. 1. Note that $Y_{i}$ for a $D E$ flip flop can assume any of the three values 0,1 , and $y_{i}$, while for a $J K$ flip flop, it can assume any of the four values $0,1, y_{i}$, and $\bar{y}_{i}$. Though the $D E$ flip flop seems, theoretically, less versatile than the $J K$ flip flop, it is by no means inferior to it within the modern circuits in which it is used such as an FPGA, in which a look-up table (LUT) is used rather than conventional logic gates ${ }^{[9-12]}$.

\section{Equational and Implicational Descriptions}

In this section, we use the Modern Syllogistic Method (MSM) ${ }^{[13-23]}$ to ferret out from the characteristic Equation (4) (viewed as a premise) all that can be concluded from it, with the resulting conclusions cast in the simplest or most compact form. A similar study was conducted in ${ }^{[1]}$ for the JK flip flop.

First, we reformulate (4) as an equation whose R.H.S. is 0, i.e.,

$f=f\left(Y_{i}, y_{i} ; D_{i}, E_{i}\right)=0$,

where

$$
\begin{gathered}
f=Y_{i} \oplus\left(y_{i} \bar{E}_{i} \vee D_{i} E_{i}\right) \\
=Y_{i}\left(\bar{y}_{l} \bar{E}_{l} \vee D_{l} E_{l}\right) \vee \bar{Y}_{i}\left(y_{i} \bar{E}_{i} \vee D_{i} E_{i}\right) \\
=Y_{i}\left(\bar{y}_{i} \bar{E}_{i} \vee \bar{D}_{i} E_{i}\right) \vee \bar{Y}_{i}\left(y_{i} \bar{E}_{i} \vee D_{i} E_{i}\right) .
\end{gathered}
$$

Then we replace $f$ in (7) by its complete-sum form using the Blake-Tison Method ${ }^{[13-27]}$. We note that (7) involves four variables, which are all biform. There is no consensus w.r.t. any of the three variables $Y_{i}, y_{i}$, and $D_{i}$. However, there are two consensuses $Y_{i} \bar{y}_{i} \bar{D}_{i}$ and $\bar{Y}_{i} y_{i} D_{i}$, which are obtained w.r.t. $E_{i}$. The resulting formula is absorptive (i.e., it has no term that can absorb another) ${ }^{[13]}$, and hence it represents the 
complete sum $C S(f)$ of $f$, and by virtue of (6), we obtain

$$
\begin{gathered}
C S(f)=Y_{i}\left(\bar{y}_{i} \bar{E}_{i} \vee \bar{D}_{i} E_{i} \vee \bar{y}_{i} \bar{D}_{i}\right) \vee \bar{Y}_{i}\left(y_{i} \bar{E}_{i} \vee D_{i} E_{i}\right. \\
\left.\vee y_{i} D_{i}\right)=0 .
\end{gathered}
$$

The expression in (8) is a disjunction of six terms equated to zero. This is exactly equivalent to each of the terms in (8) being individually equated to zero. These six equations (See Table 1) constitute all the propositions that can be stated about the $D E$ flip flop. However, we might use the equivalence $^{[13]}$

$$
\{A \rightarrow B\} \equiv\{A \bar{B}=0\},
$$

to convert each of the six equational statements in Table 1 to any of eight equivalent implicational forms, as shown in Table 1.

Complete information about the $D E$ flip flop is possible by using one of the nine equivalent statements given in each of the six main (major or double) rows in Table 1. Though Table 1 provides a wealth of facts about the $D E$ flip flops, many of these facts are redundant as they are deducible from other facts. In fact, only four independent statements suffice (albeit with some inconvenience occasionally) to characterize the $D E$ flip flop. By the "independence" requirement we rule out the following cases:

a) Selection of the first three successive equations $\quad Y_{i} \bar{y}_{i} \bar{E}_{i}=0, \quad Y_{i} \bar{D}_{i} E_{i}=0, \quad$ and $Y_{i} \bar{y}_{i} \bar{D}_{i}=0$ or the next three consecutive equations $\quad \bar{Y}_{i} y_{i} \bar{E}_{i}=0, \quad \bar{Y}_{i} D_{i} E_{i}=0, \quad$ and $\bar{Y}_{i} y_{i} D_{i}=0$. In each case, the third equation is simply the consensus of the former two, and is deducible from them.

b) Selection of two statements that belong to the same major double row, since the nine statements in the same major double row are simply equivalent. c) Selection of three statements that belong to the first three major rows or to the last three major rows.

The simplest characterization is naturally the characterization via the equational forms in major rows 1, 2, 4, and 5, (highlighted in blue in Table 1). These equations are deducible from the original characteristic function (7) equated to zero, and they are neutral about utility to analysis or synthesis. By contrast, there are six redundant analysis-oriented implicational characterizations (highlighted in yellow), in which the antecedents depend on the excitations and the consequents depend on the present and next states. There are also six redundant synthesis-oriented implicational characterizations (highlighted in green), in which the antecedents depend on the present and next states and the consequents depend on the excitations. Let us consider the four analysis-oriented implicational statements selected from major rows 1, 2, 4, and 5, namely

$$
\begin{aligned}
& \bar{E}_{i} \rightarrow \bar{Y}_{i} \vee y_{i}, \\
& \bar{D}_{i} E_{i} \rightarrow \bar{Y}_{i}, \\
& \bar{E}_{i} \rightarrow Y_{i} \vee \bar{y}_{i}, \\
& D_{i} E_{i} \rightarrow Y_{i} .
\end{aligned}
$$

The implicational statements (10) have the excitation $E_{i}$ and (possibly) the excitation $D_{i}$ in the antecedents of the implications, and have the next state $Y_{i}$ and (possibly) the present state $y_{i}$ in the consequents. Using techniques of Boolean reasoning, we can view Equations (10) as a precise translation of the characteristic map of the DE flip flop (Figs. 1(b) and 1(d)). The implication $\bar{D}_{i} E_{i} \rightarrow \bar{Y}_{i}$ in (10b) is equivalent to the second column in Fig. 1(b) or Fig. 1(d), which can be read as

$$
\left\{D_{i}=0, E_{i}=1\right\} \rightarrow\left\{Y_{i}=0\right\} .
$$


Note that this implication keeps silent about $y_{i}$. which is its way of saying that $y_{i}$ is a don't care and could be either 0 or 1 , when $D_{i}=0$ and $E_{i}=1$. Likewise, the implication $D_{i} E_{i} \rightarrow Y_{i}$ in (10d) is equivalent to the third column in Fig. 1(b) or Fig. 1(d), which can be read as

$$
\left\{D_{i}=1, E_{i}=1\right\} \rightarrow\left\{Y_{i}=1\right\} .
$$

Finally, the two implications in (10a) and (10d) can be combined as

$$
\begin{array}{r}
\left\{E_{i}=0\right\} \rightarrow\left\{\left(\bar{Y}_{i} \vee y_{i}\right)\left(Y_{i} \vee \bar{y}_{i}\right)=1\right\} \\
=\left\{\left(y_{i} \equiv Y_{i}\right)=1\right\},
\end{array}
$$

which is equivalent to the first and fourth columns combined in Fig. 1(b) or Fig. 1(d).

We now consider the four synthesis-oriented implicational statements selected from major rows $1,2,4$, and 5, namely

$$
\begin{aligned}
& Y_{i} \bar{y}_{i} \rightarrow E_{i}, \\
& Y_{i} \rightarrow D_{i} \vee \bar{E}_{i}, \\
& \bar{Y}_{i} y_{i} \rightarrow E_{i}, \\
& \bar{Y}_{i} \rightarrow \bar{D}_{i} \vee \bar{E}_{i} .
\end{aligned}
$$

The implications in (14) are the converses of those in (10) and hence they have antecedents involving the next state $Y_{i}$ and (possibly) the present state $y_{i}$ and consequents involving the excitation $E_{i}$ and (possibly) the excitation $D_{i}$. The implications in (14) are precisely equivalent to the excitation map of the $D E$ flip flop (Fig. 3(b)). The conditions (14b) and (14d) might be expanded as

$$
\begin{aligned}
& Y_{i} \rightarrow \bar{D}_{i} \bar{E}_{i} \vee D_{i} \bar{E}_{i} \vee D_{i} E_{i}, \\
& \bar{Y}_{i} \rightarrow \bar{D}_{i} \bar{E}_{i} \vee \bar{D}_{i} E_{i} \vee D_{i} \bar{E}_{i} .
\end{aligned}
$$

These mean that if $Y_{i}=1$ then $\left(D_{i}, E_{i}\right)$ belongs to $S_{1}=\{(0,0),(1,0),(1,1)\}=$ $\{(d, 0),(1,1)\}$. The condition (14a) means that if further to $Y_{i}=1$, we have $y_{i}=0$ then $E_{i}=1$, and hence $S_{1}$ reduces to $\{(1,1)\}$, i.e., $D_{i}=1$. If $Y_{i}=0$ then $\left(D_{i}, E_{i}\right)$ belongs to $S_{2}=\{(0,0),(0,1),(1,0)\}=\{(0, d),(d, 0)\}$.
The condition (14c) means that if further to $Y_{i}=0$, we have $y_{i}=1$ then $E_{i}=1$, and hence $S_{2}$ reduces to $\{(0,1)\}$, i.e., $D_{i}=0$. These arguments suffice (albeit with difficulty) to verify all entries in the two excitation maps of Fig. 3(b). It would have been more convenient if we complete the picture by augmenting Equations (14) by the two synthesis-oriented implicational statements in major rows 3 and 6, namely

$$
\begin{aligned}
& Y_{i} \bar{y}_{i} \rightarrow D_{i}, \\
& \bar{Y}_{i} y_{i} \rightarrow \bar{D}_{i} .
\end{aligned}
$$

\section{Boolean-Equation Solving for Excitations}

In this section, we employ methods of Boolean-equation solving ${ }^{[27-35]}$ to obtain all possible solutions for the excitations of a $D E$ flip flop in terms of its present state and next state. We consider a single flip flop $i$ and seek solutions of its excitations $D_{i}$ and $E_{i}$ in terms of its present state $y_{i}$ and next state $Y_{i}$. The characteristic equation of this flip flop (4) is our starting point. To solve for $D_{i}$ and $E_{i}$ in terms of $Y_{i}$ and $y_{i}$, we first convert (4) to the form of a single equation of a function equated to 1 , i.e., to the complement of (6), namely

$$
g\left(Y_{i}, y_{i} ; D_{i}, E_{i}\right)=1 \text {, }
$$

where $g$ is the complement of $f$ in (7), and is given by

$$
\begin{aligned}
g=Y_{i} \odot\left(y_{i} \bar{E}_{i} \vee\right. & \left.D_{i} E_{i}\right) \\
& =Y_{i}\left(y_{i} \bar{E}_{i} \vee D_{i} E_{i}\right) \vee \bar{Y}_{i}\left(\bar{y}_{i} \bar{E}_{i} \vee \bar{D}_{i} E_{i}\right) \\
& =\left(Y_{i} y_{i} \vee \bar{Y}_{i} \bar{y}_{i}\right) \bar{D}_{i} \bar{E}_{i} \vee\left(\bar{Y}_{i}\right) \bar{D}_{i} E_{i} \\
& \vee\left(Y_{i} y_{i} \vee \bar{Y}_{i} \bar{y}_{i}\right) D_{i} \bar{E}_{i} \\
& \vee\left(Y_{i}\right) D_{i} E_{i} .
\end{aligned}
$$

The function $g$ in (16) can be viewed as $g\left(D_{i}, E_{i}\right)$ where $g: B^{2} \rightarrow B$, and $B=B_{16}=$ $F B\left(Y_{i}, y_{i}\right)$ is the free Boolean algebra with 2 generators $Y_{i}$ and $y_{i}, 2^{2}=4$ atoms given by $\bar{Y}_{i} \bar{y}_{i}, \bar{Y}_{i} y_{i}, Y_{i} \bar{y}_{i}$ and $Y_{i} y_{i}, \quad$ and $\quad 2^{4}=16$ elements. These elements can be identified as all the switching functions of the two variables $Y_{i}$ and $y_{i}$. Figure $4(a)$ is the natural (also 
called variable-entered) map for $g\left(D_{i} . E_{i}\right)$. Figure 4(b) is a replica of Fig. 4(a) with its map entries being expanded as disjunctions of minterms of $Y_{i}$ and $y_{i}$ (i.e., as atoms of $F B\left(Y_{i}, y_{i}\right)$ ). The four atoms $\bar{Y}_{i} \bar{y}_{i}, \bar{Y}_{i} y_{i}, Y_{i} \bar{y}_{i}$, and $Y_{i} y_{i}$ make their appearances in the cells of the map of Fig. 4(b) $3,1,1$, and 3 times, respectively. Since none of the four atoms is absent in Fig. 4(b), the consistency condition for Equation (15) is satisfied trivially as an identity $\{0=0\}$. This means that Equation (15) is unconditionally consistent. It has a number of particular solutions equal to $3 * 1 * 1 * 3=9^{[1,30,32,33]}$.

Figure 4(b) can be used to construct the auxiliary function $G\left(D_{i}, E_{i}, \boldsymbol{p}\right)$ in Fig. 5. Each of the appearances of the four atoms $\bar{Y}_{i} \bar{y}_{i}, \bar{Y}_{i} y_{i} . Y_{i} \bar{y}_{i}$ and $Y_{i} y_{i}$ in Fig. 4(b), is appended in Fig. 5 by a distinguishing binary tag selected from the orthonormal sets $\left\{\bar{p}_{1} \bar{p}_{2}, \bar{p}_{1} p_{2}, p_{1}\right\},\{1\},\{1\},\left\{\bar{p}_{3} \bar{p}_{4}, \bar{p}_{3} p_{4}, p_{3}\right\}$, respectively ${ }^{[32,33]}$. For example, Fig. 5 shows the atom $\bar{Y}_{i} \bar{y}_{i}$ appended (ANDed with) $\bar{p}_{1} \bar{p}_{2}$ in the cell $\bar{D}_{i} \bar{E}_{i}$, appended with $\bar{p}_{1} p_{2}$ in the cell $D_{i} \bar{E}_{i}$, and appended with $p_{1}$ in the cell $\bar{D}_{i} E_{i}$.. Finally the solution for $D_{i}$ and $E_{i}$ is written as $[32,33]$

$D_{i}=$ Disjunction of entries of the domain $D_{i}$ in Fig. 5

$$
\begin{aligned}
& =\bar{Y}_{i} \bar{y}_{i} \bar{p}_{1} p_{2} \vee Y_{i} y_{i} \bar{p}_{3} p_{4} \vee Y_{i} \bar{y}_{i}(1) \\
& \vee Y_{i} y_{i} p_{3} \\
& =\bar{y}_{i} \bar{p}_{1} p_{2} \vee Y_{i} \bar{y}_{i} \vee Y_{i} y_{i}\left(p_{3} \vee p_{4}\right) .
\end{aligned}
$$

$E_{i}=$ Disjunction of entries of the domain $E_{i}$ in Fig. 5

$$
\begin{aligned}
=\bar{Y}_{i} \bar{y}_{i} p_{1} \vee \bar{Y}_{i} y_{i}(1) \vee Y_{i} \bar{y}_{i}(1) \vee Y_{i} y_{i} p_{3} \\
=\bar{y}_{i} p_{1} \vee \bar{Y}_{i} y_{i} \vee Y_{i} \bar{y}_{i} \vee y_{i} p_{3} .
\end{aligned}
$$

Equations (17) are a parametric solution for $D_{i}$ and $E_{i}$, where each of the four independent parameters $p_{1}, p_{2}, p_{3}$ and $p_{4}$ belongs to $B_{2}=\{0.1\}$, and hence (17) can be used to deduce the nine particular solutions of (15). These nine particular solutions for $D_{i}$ and $E_{i}$ in terms of $Y_{i}$ and $y_{i}$ are shown in algebraic and map forms in Figs. 6 and 7, respectively.
We might elect to replace the two parameters $p_{3}$ and $p_{4}$ by the two parameters $p_{1}$ and $p_{2}$ (provided we let these parameters $p_{1}$ and $p_{2}$ belong to the underlying Boolean algebra $\left.\left(B_{16}=F B\left(Y_{i}, y_{i}\right)\right)\right)$. In this case, the solution becomes

$$
\begin{aligned}
D_{i} & =\bar{y}_{i} \bar{p}_{1} p_{2} \vee Y_{i} \bar{y}_{i} \vee Y_{i} y_{i}\left(p_{1} \vee p_{2}\right), \\
E_{i} & =p_{1} \vee \bar{Y}_{i} y_{i} \vee Y_{i} \bar{y}_{i} .
\end{aligned}
$$

Equations (18) are another parametric solution for (15) that uses a minimum number of parameters (two) belonging to $B_{16}=$ $F B\left(Y_{i}, y_{i}\right)$. The solutions (17) and (18) are equivalent, since they produce the same set of nine particular solutions shown in Figs. 7 and 8.

An alternative way to solve for $D_{i}$ and $E_{i}$ is to use the concept of atomic decomposition ${ }^{[35]}$. Figures 8(a) and 8(b) present the atomic decompositions of the variables $D_{i}$ and $E_{i}$, namely

$$
\begin{aligned}
D_{i} & =\left(D_{i 0}\right) \bar{Y}_{i} \bar{y}_{i} \vee\left(D_{i 1}\right) \bar{Y}_{i} y_{i} \vee\left(D_{i 2}\right) Y_{i} \bar{y}_{i} \vee\left(D_{i 3}\right) Y_{i} y_{i},(19 a) \\
E_{i} & =\left(E_{i 0}\right) \bar{Y}_{i} \bar{y}_{i} \vee\left(E_{i 1}\right) \bar{Y}_{i} y_{i} \vee\left(E_{i 2}\right) Y_{i} \bar{y}_{i} \vee\left(E_{i 3}\right) Y_{i} y_{i},(19 b)
\end{aligned}
$$

where the four atomic components of $D\left(D_{i 0}\right.$, $\left.D_{i 1}, D_{i 2}, D_{i 3}\right)$ and those of $E\left(E_{i 0}, E_{i 1}, E_{i 2}\right.$, $\left.E_{i 3}\right)$ are arbitrary binary values. Substituting these decompositions into (16) we obtain the atomic decomposition of $g\left(D_{i}, E_{i}\right)$ as

$$
\begin{aligned}
& g\left(D_{i} . E_{i}\right)=\left(\bar{E}_{i} \vee\right.\left.\bar{D}_{i} E_{i}\right) \bar{Y}_{i} \bar{y}_{i} \vee\left(\bar{D}_{i} E_{i}\right) \bar{Y}_{i} y_{i} \vee\left(D_{i} E_{i}\right) Y_{i} \bar{y}_{i} \\
& \vee\left(\bar{E}_{i} \vee D_{i} E_{i}\right) Y_{i} y_{i} \\
&=\left(\left(\bar{E}_{i 0} \vee \bar{D}_{i 0} E_{i 0}\right)\right. \bar{Y}_{i} \bar{y}_{i} \vee\left(\bar{E}_{i 1} \vee \bar{D}_{i 1} E_{i 1}\right) \bar{Y}_{i} y_{i} \\
& \vee\left(\bar{E}_{i 2} \vee \bar{D}_{i 2} E_{i 2}\right) Y_{i} \bar{y}_{i} \\
&\left.\vee\left(\bar{E}_{i 3} \vee \bar{D}_{i 3} E_{i 3}\right) Y_{i} y_{i}\right) \bar{Y}_{i} \bar{y}_{i} \\
& \vee\left(\left(\bar{D}_{i 0} E_{i 0}\right) \bar{Y}_{i} \bar{y}_{i} \vee\left(\bar{D}_{i 1} E_{i 1}\right) \bar{Y}_{i} y_{i}\right. \\
&\left.\vee\left(\bar{D}_{i 2} E_{i 2}\right) Y_{i} \bar{y}_{i} \vee\left(\bar{D}_{i 3} E_{i 3}\right) Y_{i} y_{i}\right) \bar{Y}_{i} y_{i} \\
& \vee\left(\left(D_{i 0} E_{i 0}\right) \bar{Y}_{i} \bar{y}_{i} \vee\left(D_{i 1} E_{i 1}\right) \bar{Y}_{i} y_{i}\right. \\
&\left.\vee\left(D_{i 2} E_{i 2}\right) Y_{i} \bar{y}_{i} \vee\left(D_{i 3} E_{i 3}\right) Y_{i} y_{i}\right) Y_{i} \bar{y}_{i} \\
& \vee\left(\left(\left(\bar{E}_{i 0} \vee D_{i 0} E_{i 0}\right)\right) \bar{Y}_{i} \bar{y}_{i}\right. \\
& \vee\left(\left(\bar{E}_{i 1} \vee D_{i 1} E_{i 1}\right)\right) \bar{Y}_{i} y_{i} \\
& \vee\left(\left(\bar{E}_{i 2} \vee D_{i 2} E_{i 2}\right)\right) Y_{i} \bar{y}_{i} \\
&\left.\vee\left(\left(\bar{E}_{i 3} \vee D_{i 3} E_{i 3}\right)\right) Y_{i} y_{i}\right) Y_{i} y_{i} \\
&=\left(\bar{E}_{i 0} \vee \bar{D}_{i 0} E_{i 0}\right) \bar{Y}_{i} \bar{y}_{i} \vee\left(\bar{D}_{i 1} E_{i 1}\right) \bar{Y}_{i} y_{i} \vee\left(D_{i 2} E_{i 2}\right) Y_{i} \bar{y}_{i} \\
& \vee\left(\bar{E}_{i 3} \vee D_{i 3} E_{i 3}\right) Y_{i} y_{i}
\end{aligned}
$$


Figure 8(c) illustrates the atomic decomposition of $g\left(D_{i}, E_{i}\right)$ as given by (20). The equation to be solved forces each individual entry in the map of Fig. 8(c) to be 1 . Hence, we obtain

$$
\begin{aligned}
& \bar{E}_{i 0} \vee \bar{D}_{i 0} E_{i 0}=1, \\
& \bar{D}_{i 1} E_{i 1}=1, \\
& D_{i 2} E_{i 2}=1, \\
& \bar{E}_{i 3} \vee D_{i 3} E_{i 3}=1 .
\end{aligned}
$$

The solutions of Equations (21) are precisely those reported in Fig. 7. For convenience, we follow Brown [36] in expressing the parametric solutions (18) for $D_{i}$ and $E_{i}$ in terms of Boolean quotients (See Appendix A), namely

$$
\begin{aligned}
& D_{i}=\left(D_{i} / \bar{y}_{i}\right) \bar{y}_{i} \vee\left(D_{i} / y_{i}\right) y_{i}, \\
& E_{i}=\left(E_{i} / \bar{y}_{i}\right) \bar{y}_{i} \vee\left(E_{i} / y_{i}\right) y_{i} .
\end{aligned}
$$

$$
\begin{aligned}
D_{i} / \bar{y}_{i} & =\bar{p}_{1} p_{2} \vee Y_{i}, \\
D_{i} / y_{i} & =Y_{i}\left(p_{1} \vee p_{2}\right), \\
E_{i} / \bar{y}_{i} & =p_{1} \vee Y_{i}, \\
E_{i} / y_{i} & =p_{1} \vee \bar{Y}_{i} .
\end{aligned}
$$

\section{Conclusions}

This paper is a tutorial exposition about two widely used two-input types of flip flops, namely, $D E$ and $J K$ flip flops, with a stress on the $D E$ type. The paper contributes two novel characterizations of $D E$ flip flops, using the methods of logic deduction and Booleanequation solving. The immediate benefit to be gained from this paper is that it might help facilitate the analysis and synthesis of

\begin{tabular}{|c|c|c|c|c|}
\hline Equational form & \multicolumn{4}{|c|}{ Implicational form } \\
\hline \multirow{2}{*}{$Y_{i} \bar{y}_{i} \bar{E}_{i}=0$} & $Y_{i} \bar{y}_{i} \bar{E}_{i} \rightarrow 0$ & $Y_{i} \bar{y}_{i} \rightarrow E_{i}$ & $Y_{i} \bar{E}_{i} \rightarrow y_{i}$ & $\bar{y}_{i} \bar{E}_{i} \rightarrow \bar{Y}_{i}$ \\
\hline & $Y_{i} \rightarrow y_{i} \vee E_{i}$ & $\bar{y}_{l} \rightarrow \bar{Y}_{i} \vee E_{i}$ & $\bar{E}_{l} \rightarrow \bar{Y}_{i} \vee y_{i}$ & $1 \rightarrow \bar{Y}_{i} \vee y_{i} \vee E_{i}$ \\
\hline \multirow{2}{*}{$Y_{i} \bar{D}_{i} E_{i}=0$} & $Y_{i} \bar{D}_{i} E_{i} \rightarrow 0$ & $Y_{i} \bar{D}_{i} \rightarrow \bar{E}_{i}$ & $Y_{i} E_{i} \rightarrow D_{i}$ & $\bar{D}_{i} E_{i} \rightarrow \bar{Y}_{i}$ \\
\hline & $Y_{i} \rightarrow D_{i} \vee \bar{E}_{i}$ & $\bar{D}_{i} \rightarrow \bar{Y}_{i} \vee \bar{E}_{i}$ & $E_{i} \rightarrow \bar{Y}_{i} \vee D_{i}$ & $1 \rightarrow \bar{Y}_{i} \vee D_{i} \vee \bar{E}_{i}$ \\
\hline \multirow{2}{*}{$Y_{i} \bar{y}_{i} \bar{D}_{i}=\mathbf{0}$} & $Y_{i} \bar{y}_{i} \bar{D}_{i} \rightarrow 0$ & $Y_{i} \bar{y}_{i} \rightarrow D_{i}$ & $Y_{i} \bar{D}_{i} \rightarrow y_{i}$ & $\bar{y}_{i} \bar{D}_{i} \rightarrow \bar{Y}_{i}$ \\
\hline & $Y_{i} \rightarrow y_{i} \vee D_{i}$ & $\bar{y}_{i} \rightarrow \bar{Y}_{i} \vee D_{i}$ & $\bar{D}_{i} \rightarrow \bar{Y}_{i} \vee y_{i}$ & $1 \rightarrow \bar{Y}_{i} \vee y_{i} \vee D_{i}$ \\
\hline \multirow{2}{*}{$\bar{Y}_{i} y_{i} \bar{E}_{i}=0$} & $\bar{Y}_{i} y_{i} \bar{E}_{i} \rightarrow 0$ & $\bar{Y}_{i} y_{i} \rightarrow E_{i}$ & $\bar{Y}_{i} \bar{E}_{i} \rightarrow \bar{y}_{i}$ & $y_{i} \bar{E}_{i} \rightarrow Y_{i}$ \\
\hline & $\bar{Y}_{i} \rightarrow \bar{y}_{i} \vee E_{i}$ & $y_{i} \rightarrow Y_{i} \vee E_{i}$ & $\bar{E}_{l} \rightarrow Y_{i} \vee \bar{y}_{i}$ & $1 \rightarrow Y_{i} \vee \bar{y}_{i} \vee E_{i}$ \\
\hline \multirow{2}{*}{$\bar{Y}_{t} D_{i} E_{i}=0$} & $\bar{Y}_{l} D_{i} E_{i} \rightarrow 0$ & $\bar{Y}_{i} D_{i} \rightarrow \bar{E}_{i}$ & $\bar{Y}_{l} E_{i} \rightarrow \bar{D}_{i}$ & $D_{i} E_{i} \rightarrow Y_{i}$ \\
\hline & $\bar{Y}_{i} \rightarrow \bar{D}_{i} \vee \bar{E}_{i}$ & $D_{i} \rightarrow Y_{i} \vee \bar{E}_{i}$ & $E_{i} \rightarrow Y_{i} \vee \bar{D}_{i}$ & $1 \rightarrow Y_{i} \vee \bar{D}_{i} \vee \bar{E}_{i}$ \\
\hline \multirow{2}{*}{$\bar{Y}_{i} y_{i} D_{i}=0$} & $\bar{Y}_{i} y_{i} D_{i} \rightarrow 0$ & $\bar{Y}_{i} y_{i} \rightarrow \bar{D}_{i}$ & $\bar{Y}_{i} D_{i} \rightarrow \bar{y}_{i}$ & $y_{i} D_{i} \rightarrow Y_{i}$ \\
\hline & $\bar{Y}_{i} \rightarrow \bar{y}_{i} \vee \bar{D}_{i}$ & $y_{i} \rightarrow Y_{i} \vee \bar{D}_{i}$ & $D_{i} \rightarrow Y_{i} \vee \bar{y}_{i}$ & $1 \rightarrow Y_{i} \vee \bar{y}_{i} \vee \bar{D}_{i}$ \\
\hline
\end{tabular}
sequential digital circuits. Future work in support and continuation of the present analysis might include the provision of some simulation and implementation results.

Here, the Boolean quotients are

Table 1. All possible statements that can be made about $D E$ flip flops (arranged in six major double rows). Four irredundant equational characterizations are highlighted in blue. Six redundant analysis-oriented implicational characterizations are highlighted in yellow. Six redundant synthesis-oriented implicational characterizations are highlighted in green. 


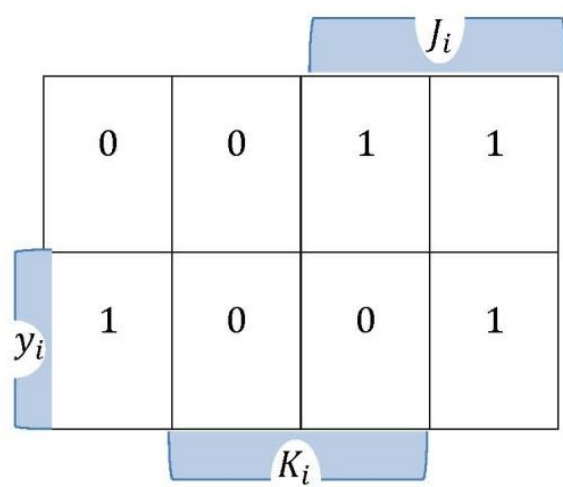

$Y_{i}$

(a) The $J K$ flip flop (conventional map)

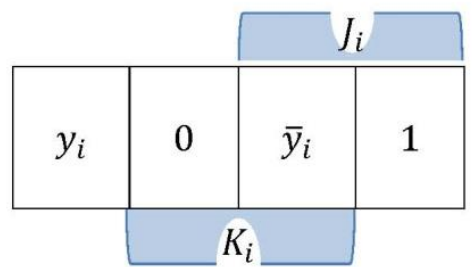

$Y_{i}$

(c) The $J K$ flip flop (with present state as entered variable)

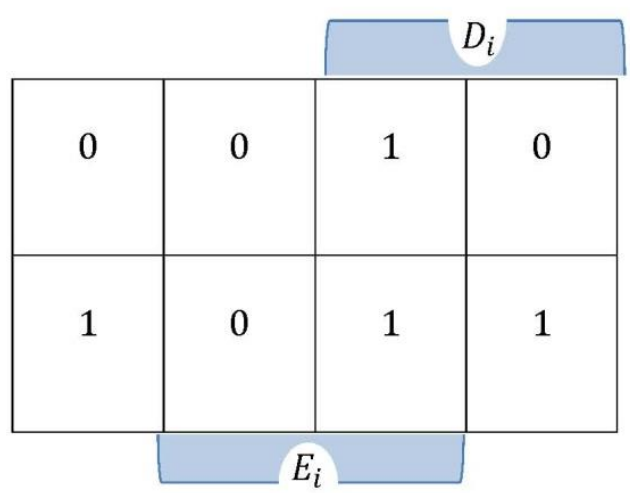

$Y_{i}$

(b) The $D E$ flip flop (conventional map)

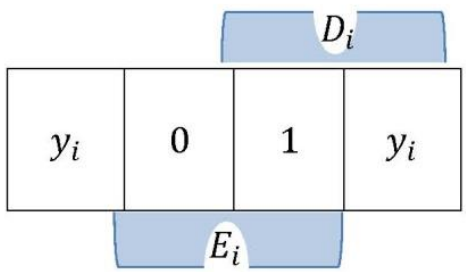

$Y_{i}$

(d) The $D E$ flip flop (with present state as entered variable)

Fig. 1. Characteristic maps for the JK and DE flip flops.

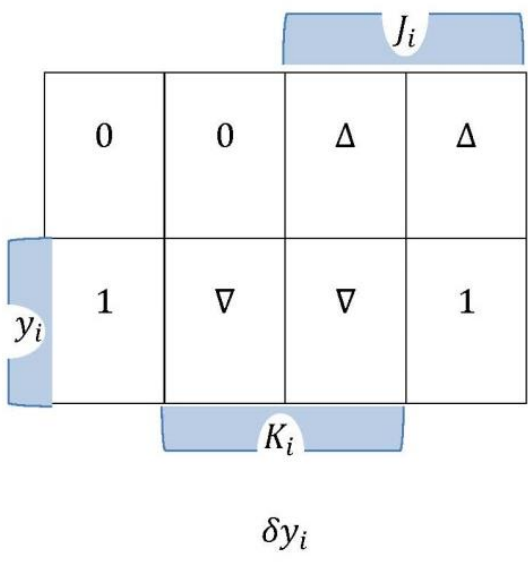

(a) The $J K$ flip flop

\begin{tabular}{|l|l|l|l|}
\cline { 3 - 4 } \multicolumn{2}{c|}{} & \multicolumn{2}{c|}{$D_{i}$} \\
\hline 0 & 0 & $\Delta$ & 0 \\
\hline 1 & $\nabla$ & 1 & 1 \\
\hline & & & \\
\hline
\end{tabular}

$\delta y_{i}$

(b) The $D E$ flip flop

Fig. 2. Transition maps for the JK and DE flip flops. 


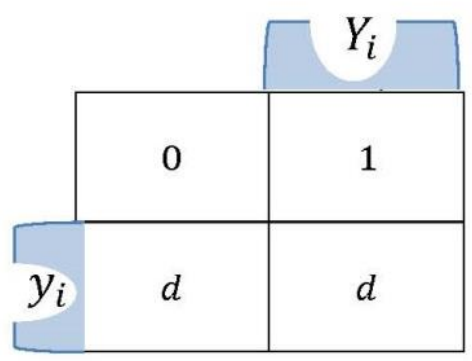

$J_{i}$

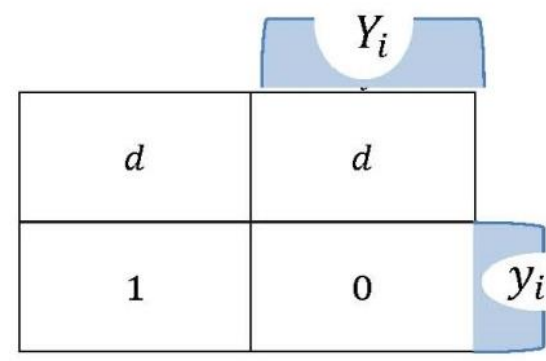

$K_{i}$

(a) The JK flip flop

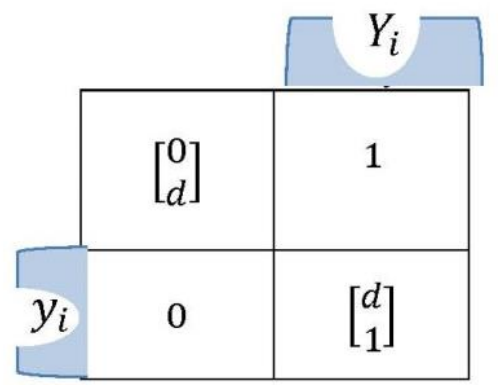

$D_{i}$

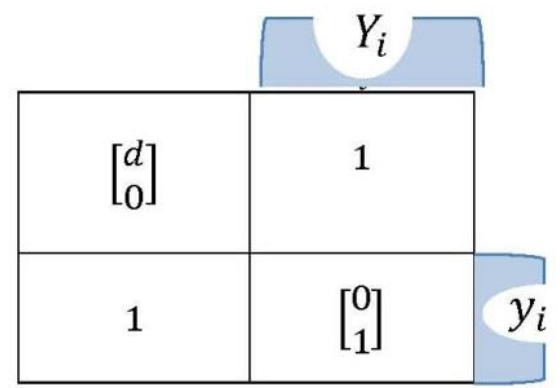

$E_{i}$

(b) The DE flip flop

Fig. 3. Excitation maps for the JK and DE flip flops. 


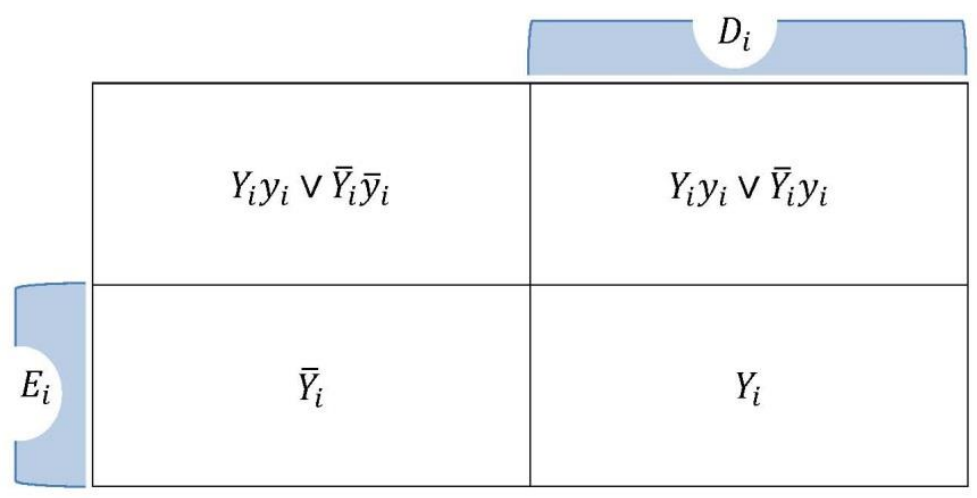

(a) $g\left(D_{i}, E_{i}\right)$

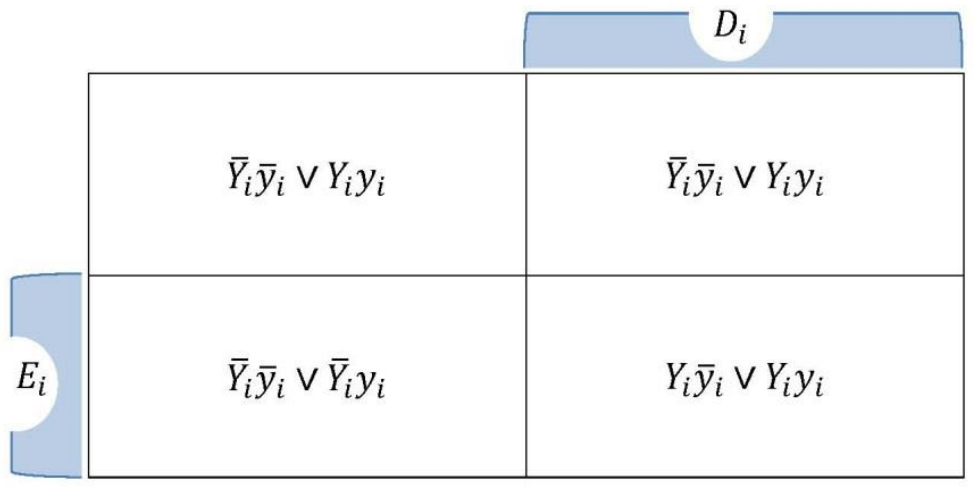

(b) $g\left(D_{i}, E_{i}\right)$

Fig. 4. Natural map for the function $g\left(D_{i}, E_{i}\right)$ as obtained from (16) and with minterm-expanded entries.

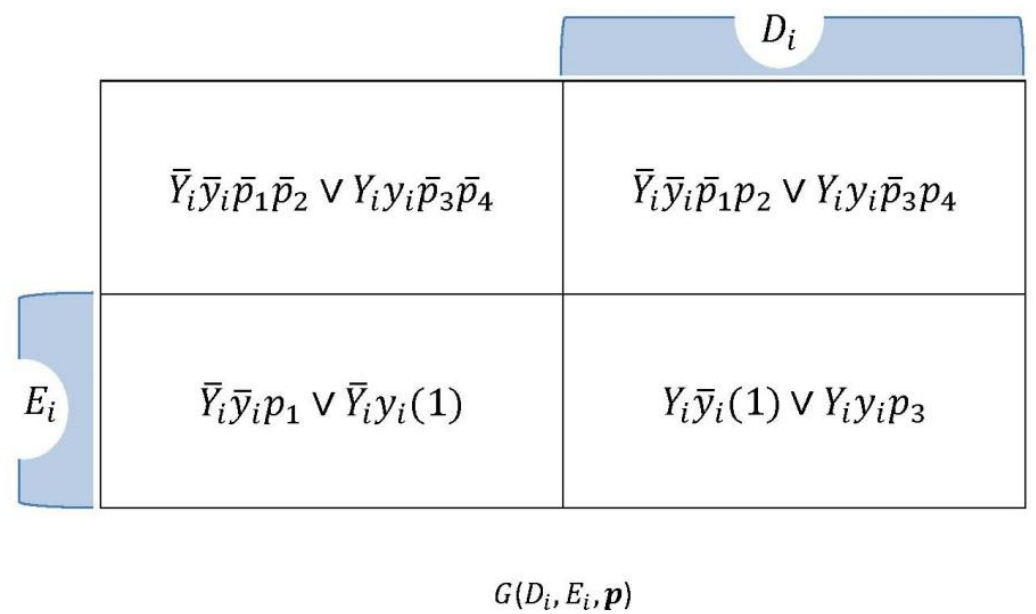

Fig. 5. Natural map for the auxiliary function $G\left(D_{i}, E_{i}, p\right)$. 


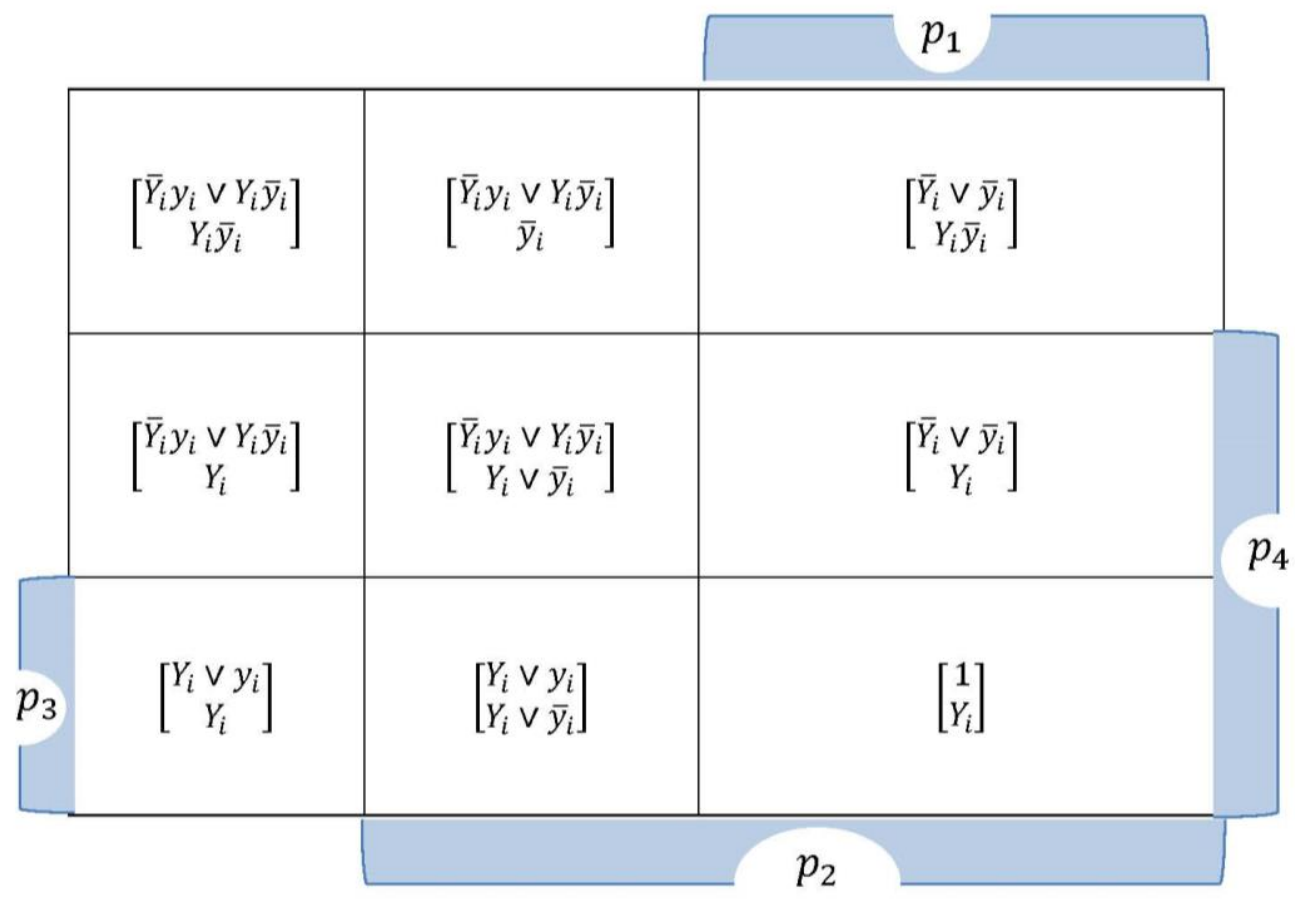

$\left[\begin{array}{c}E_{i} \\ D_{i}\end{array}\right]$

Fig. 6. The nine particular solutions for $E_{i}$ and $D_{i}$ expressed algebraically in terms of $Y_{i}$ and $y_{i}$. One of these solutions $\left(E_{i}=1, D_{i}=Y_{i}\right)$ asserts that upon enabling, the DE flip flop behaves as a standard D flip flop.
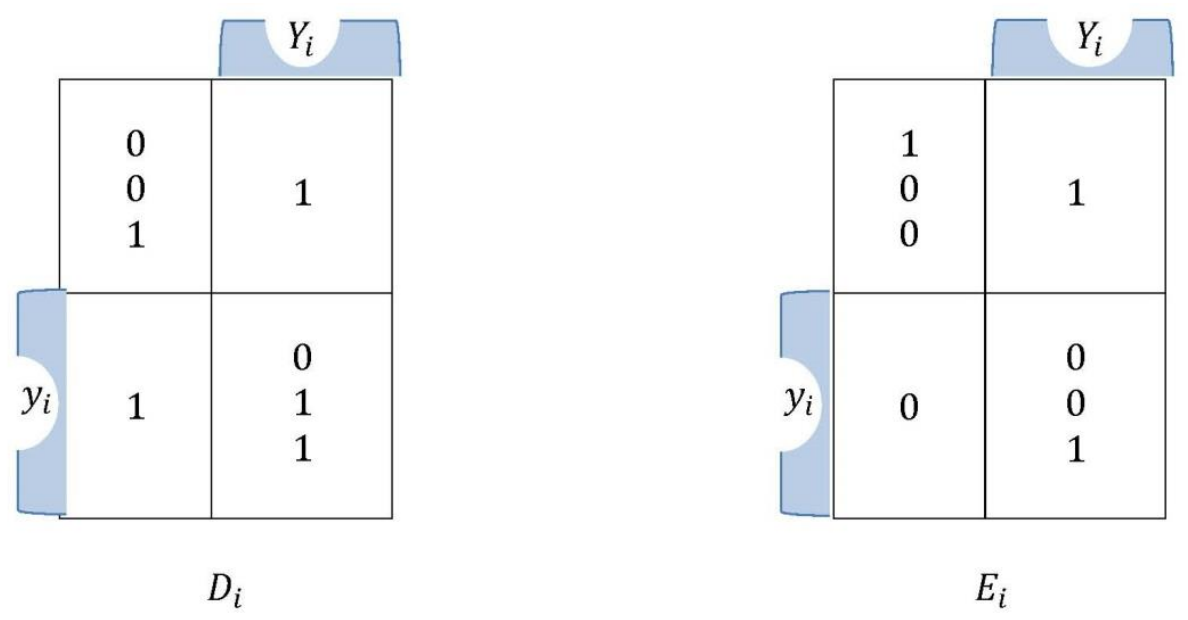

Fig. 7. The nine particular solutions for $D_{i}$ and $E_{i}$ expressed in terms of maps of map variables $Y_{i}$ and $y_{i}$. This figure is an expanded version of the excitation map of the $D E$ flip flop in Fig. 3(b). 


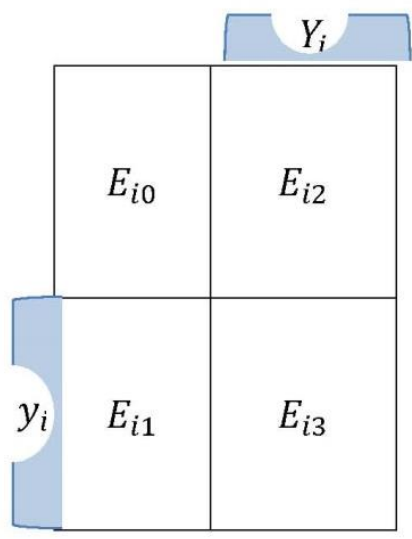

(a) $E_{i}$

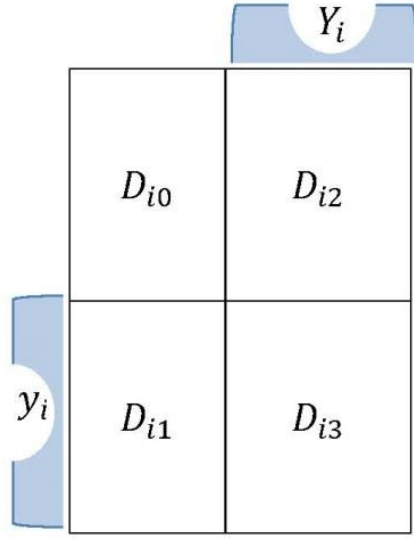

(b) $D_{i}$

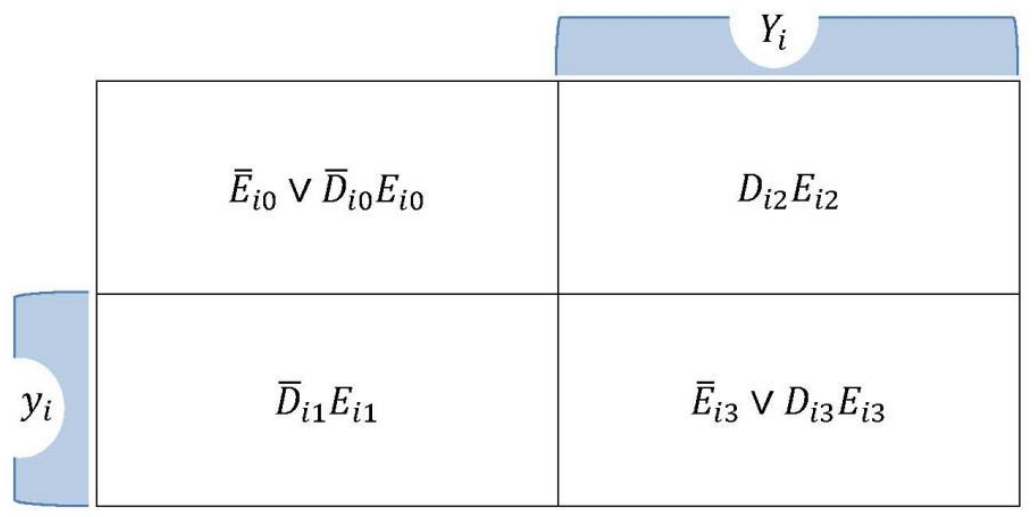

(c) $g\left(D_{i}, E_{i}\right)$

Fig. 8. Atomic decompositions of the variables $D_{i}$ and $E_{i}$ as well as of the function $g\left(D_{i}, E_{i}\right)$ equated to 1 .

\section{References}

[1] Rushdi, A. M. A. and Ghaleb, F. A. M., Novel Characterizations of the JK Bistables (Flip Flops), Journal of Engineering Research and Reports, 4(3): 1-20 (2019).

[2] Lee, S. C., Modern Switching Theory and Digital Design, Prentice-Hall, Englewood Cliffs, New Jersey, NJ, USA (1978).

[3] Muroga, S., Logic Design and Switching Theory, John Wiley \& Sons, New York, NY, US (1979).

[4] Fletcher, W. I., An Engineering Approach to Digital Design, Prentice-Hall, Englewood Cliffs, New Jersey, NJ, USA (1980).

[5] Unger, S. H., The Essence of Logic Circuits. PrenticeHall, Englewood Cliffs, New Jersey, NJ, USA (1989).
[6] Hill, F. J. and Peterson, G. R., Computer Aided Logical Design with Emphasis on VLSI, 4th Edition, John Wiley \& Sons, New York, NY, USA (1993).

[7] Vingron, S. P., Logic Circuit Design: Selected Methods, pp. 51-66. Springer-Verlag, Berlin-Heidelberg, Germany (2012).

[8] Wang, C. and Tao, Y., Karnaugh maps of logical systems and applications in digital circuit design. Circuits, Systems, and Signal Processing, 1-27 (2019).

[9] Tucker, J. H. and Tapia, M. A., Generalized flip-flop input equations based on a four-valued Boolean algebra. In Proceedings IEEE SOUTHEASTCON'97, IEEE: 82-86 (1997, April).

[10] Brown, S. and Rose, J., FPGA and CPLD architectures: A tutorial. IEEE Design \& Test of Computers, 13(2): 4257 (1996). 
[11] Kaeslin, H., Digital Integrated Circuit Design: From VLSI Architectures to CMOS Fabrication, Cambridge University Press (2008).

[12] Donzellini, G., Oneto, L., Ponta, D. and Anguita, D., Introduction to Digital Systems Design. Springer, Cham (2019).

[13] Brown, F. M., Boolean Reasoning: The Logic of Boolean Equations, Kluwer Academic Publishers, Boston, MA, USA (1990).

[14] Rushdi, A. M. and Ba-Rukab, O. M., An exposition of the modern syllogistic method of propositional logic. Umm Al-Qura University Journal: Engineering and Architecture, 1(1): 17-49 (2009).

[15] Rushdi, A. M. and Ba-Rukab, O. M., Map derivation of the closures for dependency and attribute sets and all candidate keys for a relational database. Journal of King Abdulaziz University: Engineering Sciences, 25(2): 3-33 (2014).

[16] Rushdi, A. M., Zarouan, M., Alshehri, T. M. and Rushdi, M. A., A modern syllogistic method in intuitionistic fuzzy logic with realistic tautology. The Scientific World Journal, 2015, Article ID 327390, 12 pages (2015).

[17] Rushdi, A. M., Alshehri, T. M., Zarouan, M. and Rushdi, M. A., Utilization of the modern syllogistic method in the exploration of hidden aspects in engineering ethical dilemmas. Journal of King Abdulaziz University: Computers and Information Technology, 3(1): 73-127 (2015).

[18] Rushdi, A. M., Zarouan, M., Alshehri, T. M. and Rushdi, M. A., The incremental version of the modern syllogistic method. Journal of King Abdulaziz University: Engineering Sciences, 26(1): 25-54 (2015).

[19] Rushdi, A. M. and Rushdi, M. A., Utilization of the modern syllogistic method in the service of academic advising. In Proceedings of KAU Conference on Academic Advising in Higher Education, 228-241 (2015).

[20] Rushdi, A. M. and Rushdi, M. A., Switching-algebraic algorithmic derivation of candidate keys in relational databases. In 2016 International IEEE Conference on Emerging Trends in Communication Technologies (ETCT), 1-5 (2016, November).

[21] Rushdi, A. M. and Rushdi, M. A., Mathematics and Examples of the Modern Syllogistic Method of Propositional Logic, Chapter 6 in Ram, M. (Editor), Mathematics Applied in Information Systems, Bentham Science Publishers, Emirate of Sharjah, United Arab Emirates, 123-167 (2018).

[22] Rushdi, A. M. A., Utilization of Karnaugh maps in multi-value qualitative comparative analysis, International Journal of Mathematical, Engineering and Management Sciences (IJMEMS), 3(1): 28-46 (2018).

[23] Rushdi, R. A. and Rushdi, A. M., Karnaugh-map utility in medical studies: The case of Fetal Malnutrition. International Journal of Mathematical, Engineering and Management Sciences (IJMEMS), 3(3): 220-244 (2018).
[24] Rushdi, A. M. and Al-Yahya, H. A., Derivation of the complete sum of a switching function with the aid of the variable-entered Karnaugh map. Journal of King Saud University-Engineering Sciences, 13(2): 239-268 (2001).

[25] Rushdi, A. M., Prime-implicant extraction with the aid of the variable-entered Karnaugh map. Umm Al-Qura University Journal: Science, Medicine and Engineering, 13(1): 53-74 (2001).

[26] Rushdi, A. M. and Al-Yahya, H. A., Variable-entered Karnaugh map procedures for obtaining the irredundant disjunctive forms of a switching function from its complete sum. Journal of King Saud UniversityEngineering Sciences, 14(1): 13-26 (2002).

[27] Rushdi, A. M. A. and Albarakati, H. M., Construction of general subsumptive solutions of Boolean equations via complete-sum derivation. Journal of Mathematics and statistics, 10(2): 155-168 (2014).

[28] Rushdi, A. M., Using variable-entered Karnaugh maps to solve Boolean equations. International Journal of Computer Mathematics, 78(1): 23-38 (2001).

[29] Rushdi, A. M. A., Efficient solution of Boolean equations using Variable-Entered Karnaugh Maps. King Abdulaziz University: Engineering Sciences, 15(1): 105121 (2004).

[30] Rushdi, A. M. and Amashah, M. H., Using variableentered Karnaugh maps to produce compact parametric general solutions of Boolean equations, International Journal of Computer Mathematics, 88(15): 3136-3149 (2011).

[31] Rushdi, A. M. A. and Albarakati, H. M., Prominent classes of the most general subsumptive solutions of Boolean equations. Information Sciences, 281: 53-65 (2014).

[32] Rushdi, A. M. A. and Ahmad, W., A novel method for compact listing of all particular solutions of a system of Boolean equations. British Journal of Mathematics \& Computer Science, 22(6): 1-18 (2017).

[33] Rushdi, A. M. and Zagzoog, S. S., Derivation of all particular solutions of a 'big' Boolean equation with applications in digital design. Current Journal of Applied Science and Technology, 27(3): 1-16 (2018).

[34] Bhattacharjee, P. R., A novel method for solving simultaneous equations in Boolean/switching algebra. IETE Journal of Education, 59(1): 18-25 (2018).

[35] Balamesh, A. S. and Rushdi, A. M., Solution of Boolean equations via atomic decomposition into independent switching equations, to appear ISA.

[36] Brown, F. M., Single-parameter solutions for flip-flop equations. IEEE Transactions on Computers, C-20(4): 452-454 (1971).

[37] Rushdi, A. M. and Rushdi, M. A., Switching-Algebraic Analysis of System Reliability. Chapter 6 (pp. 139-161) in M. Ram and P. Davim (Editors), Advances in Reliability and System Engineering. Cham, Switzerland: Springer International Publishing (2017).

[38] Rushdi, A. M. A., Utilization of symmetric switching functions in the symbolic reliability analysis of multi- 
state k-out-of-n systems, International Journal of Mathematical, Engineering and Management Science (IJMEMS), 4(2): 306-326 (2019).

[39] Reusch, B., Generation of prime implicants from subfunctions and a unifying approach to the covering problem. IEEE Transactions on Computers, C-24(9): 924-930 (1975).

[40] Rushdi, A. M., Improved variable-entered Karnaugh map procedures. Computers \& Electrical Engineering, 13(1): 4152 (1987).

[41] Bryant, R. E., Symbolic Boolean manipulation with ordered binary-decision diagrams. ACM Computing Surveys (CSUR), 24(3): 293-318 (1992).
[42] Rushdi, A. M. A. and Al-Qwasmi, M. A., Exposition and comparison of two kinds of a posteriori analysis of fault trees, Journal of King Abdulaziz University: Computing and Information Technology, 5(1-2): 55-74 (2016).

[43] Rushdi, R. A. and Rushdi, A. M., Common fallacies of probability in medical context: A simple mathematical exposition, Journal of Advances in Medicine and Medical Research, 26(1): 1-21 (2018).

[44] Rushdi, A. M. A. and Ghaleb, F. A. M., A tutorial exposition of semi-tensor products of matrices with a stress on their representation of Boolean functions. Journal of King Abdulaziz University: Faculty of Computers and Information Technology, 5(1-2): 3-30 (2016). 


\section{Appendix A}

\section{Boolean Quotients and the Boole-Shannon Expansion}

The concept of a Boolean quotient is a switching-algebraic concept that can be conveniently used to facilitate Boolean manipulations. Given a two-valued Boolean function $f$ and a term $t$, the Boolean quotient of $f$ with respect to $t$, denoted by $(f / t)$ or $(f \mid t)$, is defined to be the function formed from $f$ by explicitly imposing the condition $\{t=1\}$ (See Brown ${ }^{[13]}$, Rushdi and Rushdi ${ }^{[37]}$, or Rushdi $\left.{ }^{[38]}\right)$, i.e.,

$$
f / t=[f]_{t=1},
$$

The Boolean quotient is also known as a ratio ${ }^{[3]}$, a subfunction ${ }^{[39,40]}$ or a restriction ${ }^{[41]}$. An important feature of Boolean quotients is that the conjunction of a term with a function is equal to the conjunction of the term with the Boolean quotient of the function with respect to the term, viz.,

$$
t \wedge f=t \wedge(f / t) .
$$

If the term $t$ is implied by the function $f$ (i.e., $f \leq t, f \rightarrow t, f=t \wedge f$ ), then (A.2) reduces to

$$
f=t \wedge(f / t) \text {. }
$$

The concept of the Boolean quotient has a striking similarity to that of conditional probability [37, 42, 43], but perhaps the most important utilization of the Boolean quotient is its use in the Boole-Shannon Expansion, which constitutes the most fundamental theorem of Boolean algebra (See Brown ${ }^{[13]}$, Rushdi and Rushdi ${ }^{[37]}$, or Rushdi and Ghaleb ${ }^{[44]}$ )

$f(\boldsymbol{X})=\left(\bar{X}_{i} \wedge\left(f(\boldsymbol{X}) / \bar{X}_{i}\right)\right) \vee\left(X_{\mathrm{i}} \wedge\left(f(\boldsymbol{X}) / X_{\mathrm{i}}\right)\right)$, (A.4)

For example, the next state $Y_{i}$ of flip flop number $i$ can be expressed in terms of the present state $y_{i}$ of the same flip flop as

$$
Y_{i}=\left(Y_{i} / \bar{y}_{i}\right) \bar{y}_{i} \vee\left(Y_{i} / y_{i}\right) y_{i}
$$

The two Boolean quotients $\left(Y_{i} / \bar{y}_{i}\right)$ and $\left(Y_{i} / y_{i}\right)$ in (A.5) are independent of the state of flip flop $i$. They are functions of other variables of the circuit, including inputs to the excitation logic and (possibly) the present states of other flip flops. For the $D E$ flip flop, they are given by $D_{i} E_{i}$ and $\bar{E}_{i} \vee D_{i}$, respectively. 


\section{توصيفان مبتكران لبدال تمكين التأخير (م خ)}

\section{علي محمد علي رشدي و فارس أحمد محمد غالب}

قسم الهندسة الكهبائبة وهندسة الحاسبات، كلية الهندسة، جامعة الملك عبد العزيز، جلة 019 1 ، المدلكة العربية السعودية arushdi@kau.edu.sa

المستخلص. تستعمل الدوائر الرقمية الحديثة، وخاصة تلك المبنية علي النبائط ذات المكاملة

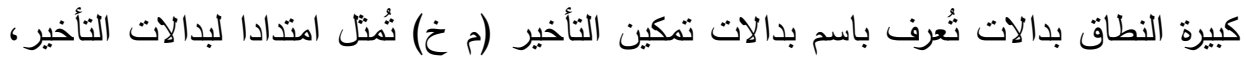
تتمتع بالمقدرة علي تخزين قيمة مدخلة بحيث يكون هذا التخزين بالطلب أو التمكين. يتم وصف هذا البدال جبريا بواسطة معادلته المميزة، أو جدوليا بواسطة جدول الحالة التالية (المستعمل بالية لأغراض التحليل) أو جدول الاستثارة (المستعمل لأغراض التركيب والاصطناع). تستكثف ورقة البحث هذه توصيفين مبتكرين لبدال تمكين التأخير (م خ). بداية، ينت وصف البدال بتوصيفات

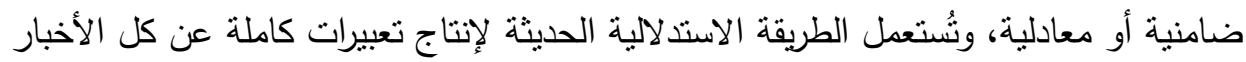
التي تصح بالنسبة لبدال تمكين التأخير • يلي ذلك توظيف طرائق حل المعادلات البولانية لإيجاد جميع الأساليب الممكنة للتعبير عن مدخلي الاستثارة (التخذية) بدلالة الحالة الراهنة والحالة التالية. وهنا يلعب مفهوم "خارج القسمة البولاني" دورا هاما في توضيح المفاهيم المعنية وفي إنجاز الاشثقاقات المطلوبة المختلفة. ويُرجي لهذه الورقة أن تكون ذات نفع نعليمي مباشر وأن تيسر تحليل وتركيب الدوائر الرقمية التتابعية. كلمات مفتاحية: البدال، التوصيف، الطريقة الاستدلالية الحديثة، حل المعادلات البولانية، خارج القسمة البولاني، الاستثارة (التغذية). 